\title{
@creative
commons
}

\section{Trees with small spectral gap}

\author{
Ivana Jovović *, Tamara Koledin \\ Faculty of Electrical Engineering, University of Belgrade, \\ Bulevar Kralja Aleksandra 73, 11000 Belgrade, Serbia \\ Zoran Stanić $^{\dagger}$ \\ Faculty of Mathematics, University of Belgrade, \\ Studentski trg 16, 11000 Belgrade, Serbia
}

Received 7 December 2015, accepted 20 April 2017, published online 25 August 2017

\begin{abstract}
Continuing the previous research, we consider trees with given number of vertices and minimal spectral gap. Using the computer search, we conjecture that this spectral invariant is minimized for double comet trees. The conjecture is confirmed for trees with at most 20 vertices; simultaneously no counterexamples are encountered. We provide theoretical results concerning double comets and putative trees that minimize the spectral gap. We also compare the spectral gap of regular graphs and paths. Finally, a sequence of inequalities that involve the same invariant is obtained.
\end{abstract}

Keywords: Graph eigenvalues, double comet, extremal values, numerical computation.

Math. Subj. Class.: 05C50, 65F15

\section{Introduction}

We use standard graph-theoretic terminology and notation. For example, for a graph $G$, $n=n(G)$ and $m=m(G)$ denote its order and size, while $A=A(G)$ stands for its adjacency matrix. The characteristic polynomial of $A$ is the characteristic polynomial of $G$, denoted $P_{G}$. Its roots are the eigenvalues of $G$, denoted $\lambda_{1}(G) \geq \lambda_{2}(G) \geq \cdots \geq \lambda_{n}(G)$. Non-isomorphic graphs that share the same spectrum are said to be cospectral.

${ }^{*}$ Research is partially supported by Serbian Ministry of Education, Science and Technological Development, Project 174032.

${ }^{\dagger}$ Research is partially supported by Serbian Ministry of Education, Science and Technological Development, Projects 174012 and 174033.

E-mail addresses: ivana@etf.rs (Ivana Jovović), tamara@etf.rs (Tamara Koledin), zstanic@matf.bg.ac.rs, zstanic@math.rs (Zoran Stanić)

(a) (i) This work is licensed under http://creativecommons.org/licenses/by/3.0/ 
The difference between the first two eigenvalues $\delta(G)=\lambda_{1}(G)-\lambda_{2}(G)$ is called the spectral gap of $G$. In the previous investigation (see [13]), graphs with small spectral gap are considered. The purpose of this paper is to continue this investigation in the case of trees (this part can be considered as counterpart to [13], as well), consider regular graphs with small spectral gap, and give certain upper and lower bounds for this spectral invariant.

As announced in the Abstract, we conjecture that a minimal spectral gap of trees with given order is attained for a double comet tree (the conjecture is formulated in the very beginning of the next section). We also give certain theoretical and computational results supporting this conjecture, and consider cospectrality of double comets. The conjecture is open, but nevertheless we give additional (structural and spectral) properties of a tree with minimal spectral gap.

Next, we compare the spectral gap of regular graphs and paths by showing that, under some restrictions, the spectral gap of a regular graph is bounded from below by the spectral gap of the corresponding path. Finally, we give some new bounds on $\delta$ expressed in terms of order, size, clique number, and minimal or maximal vertex degree.

We conclude this section by certain terminology and notation. The main results are given in Sections 2-4.

We use $P_{n}, C_{n}, K_{n}$, and $K_{n_{1}, n_{2}}\left(n_{1}+n_{2}=n\right)$ to denote the path, cycle, complete graph (or clique), and complete bipartite graph on $n$ vertices, respectively. The disjoint union of graphs $G$ and $H$ is denoted by $G \cup H$, while the disjoint union of $k$ copies of $G$ is denoted by $k G$.

The generalized double comet $C^{*}\left(k_{1}, k_{2}, l\right)$, where $k_{1}, k_{2} \geq 0$ and $l \geq 2$, is a tree obtained by attaching $k_{1}$ pendant vertices at one end of the path $P_{l}$ and $k_{2}$ pendant vertices at the other end of the same path. The path $P_{l}$ is referred to as the internal path. The double comet $C(k, l)$ is obtained from $C^{*}\left(k_{1}, k_{2}, l\right)$ for $k_{1}=k_{2}=k$.

\section{Spectral gap of trees}

We start with the following conjecture.

Conjecture 2.1. Among all trees of order n, the spectral gap is minimized for some double comet.

The conjecture is confirmed by computer search for trees with at most 20 vertices. In all situations, there is a unique tree attaining the minimal spectral gap. For $n \leq 8$, this is the path $P_{n}$ (which is a special case of double comet), for $9 \leq n \leq 11$ this is $C(2, n-4)$, for $12 \leq n \leq 15$ this is $C(3, n-6)$, and for $16 \leq n \leq 20$ this is $C(4, n-8)$.

If $\Delta$ and $\Delta^{\prime}(<\Delta)$ are the largest and second largest degree in a tree $T$ and the distance between the vertices having these degrees is at least three, then using the Interlacing Theorem (cf. [14, Theorem 1.6]), we get $\lambda_{2}(T) \geq \lambda_{1}\left(K_{1, \Delta^{\prime}}\right)=\sqrt{\Delta^{\prime}}$. In other words, $\sqrt{\Delta^{\prime}}$ makes a lower bound on $\lambda_{2}(T)$. Since an increase in $\Delta^{\prime}$ increases this bound, it is natural to assume that the spectral gap is minimized for a tree with two vertices of maximal degree. We provide more theoretical results supporting Conjecture 2.1.

Theorem 2.2. In the set of trees of order $n(n \geq 2)$ and two vertices of maximal degree $\Delta$, the largest eigenvalue $\lambda_{1}$ is minimized for the double comet $C(\Delta-1, n-2(\Delta-1))$.

Proof. Clearly, the statement holds for $\Delta \leq 2$. Assume next that $u, v$ are the two vertices of degree $\Delta(\geq 3)$ in a tree $T$ and let $N(u), N(v)$ be their open neighbourhoods. Then, 
removing a pendant vertex which does not belong to $N(u) \cup N(v)$ (if such a vertex does not exist, then $T$ is the required double comet) impose a strict decrease in $\lambda_{1}$ (as a consequence of the Perron-Frobenius Theorem, cf. [2, Theorem 1.3.6]). In addition, by the well-known result of Hoffman and Smith [4], inserting a vertex in the unique path between $u$ and $v$ is again followed by a strict decrease in $\lambda_{1}$. Therefore, rearranging a described pendant vertex into the corresponding path gives a tree with smaller largest eigenvalue. Repeating this procedure, after finite number of steps, we arrive at $C(\Delta-1, n-2(\Delta-1))$.

In the following theorem we use another result of Hoffman [3]: Let $H$ be the graph obtained from a graph $G$ by attaching a hanging path of an arbitrary length at any vertex $v$ (of $G$ ) of the degree at least two. When the length of the path attached tends to infinity then the largest eigenvalue of $H$ increases and tends to the largest root of the equation

$$
f(x)=\left(\frac{x+\sqrt{x^{2}-4}}{2}\right) P_{G}(x)-P_{G-v}(x)=0,
$$

whenever the largest eigenvalue of $H$ is greater than 2 .

Now we have the following theorem comparing spectral gaps of generalized double comets with equal internal paths.

Theorem 2.3. Given a generalized double comet $C^{*}\left(k_{1}, k_{2}, l\right)$. If $l \geq 2$ and $k_{1} \leq k_{2}-2$, then $\delta\left(C^{*}\left(k_{1}, k_{2}, l\right)\right)>\delta\left(C^{*}\left(k_{1}+1, k_{2}-1, l\right)\right)$.

Proof. Let, for short, $T$ (resp. $S$ ) stand for $C^{*}\left(k_{1}, k_{2}, l\right)$ (resp. $\left.C^{*}\left(k_{1}+1, k_{2}-1, l\right)\right)$.

We have $\lambda_{1}(T)>\lambda_{1}(S)$, as proved in [12], and so it remains to prove that $\lambda_{2}(T) \leq$ $\lambda_{2}(S)$. First, we consider the three singular cases.

For $l=2$, Using the Schwenk formula [2, Theorem 2.4.3], we compute

$$
P_{T}(x)=x^{k_{1}+k_{2}-2} Q(x) \text { and } P_{S}(x)=x^{k_{1}+k_{2}-2} R(x)
$$

where

$$
\begin{aligned}
& Q(x)=\left(x^{4}-\left(k_{1}+k_{2}+1\right) x^{2}+k_{1} k_{2}\right) \text { and } \\
& R(x)=\left(x^{4}-\left(k_{1}+k_{2}+1\right) x^{2}+k_{1} k_{2}-k_{1}+k_{2}-1\right) .
\end{aligned}
$$

The second largest eigenvalues of both trees coincide with second largest roots of the polynomials $Q$ and $R$. Since $R(x)-Q(x)=-k_{1}+k_{2}-1>0$, we get $\lambda_{2}(T)<\lambda_{2}(S)$.

For $l=3$, by removing the vertex of maximal degree in $T$ and using the interlacing argument, we get $\lambda_{2}(T) \leq \sqrt{k_{1}+1}$. Similarly, by removing the middle vertex of the internal path in $S$, we get $\lambda_{2}(S) \geq \sqrt{k_{1}+1}$, and we are done.

For $l=4$, following the case $l=2$, we get that the characteristic polynomials of trees $T$ and $S$ are $P_{T}(x)=x^{k_{1}+k_{2}-2} Q_{1}(x)$ and $P_{S}(x)=x^{k_{1}+k_{2}-2} R_{1}(x)$, where the polynomials $Q_{1}$ and $R_{1}$ are computed in a similar way, while their difference is $R_{1}(x)-Q_{1}(x)=$ $\left(k_{1}-k_{2}+1\right)\left(1-x^{2}\right)$. Computing this value for $x=\lambda_{2}(T)$, we get the assertion.

Let now $l \geq 5$. For $k_{1}=2$ we directly get $\lambda_{2}(T) \leq 2 \leq \lambda_{2}(S)$, and similarly for $k_{1}=1$. Let in further $T^{\prime}$ stand for the non-trivial component of the graph obtained by 


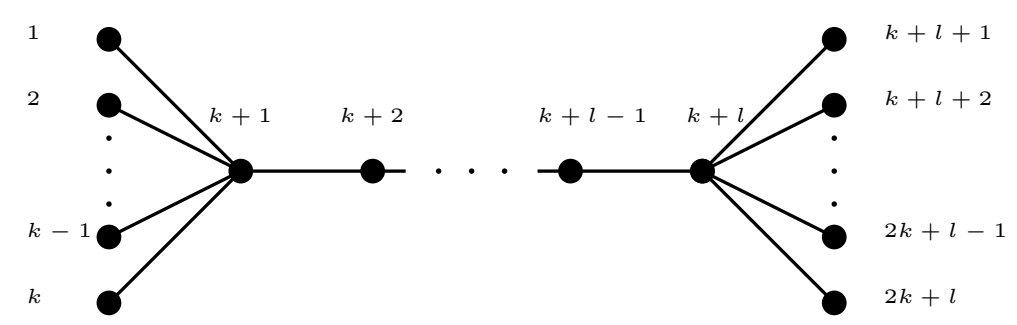

Figure 1: Double comet $C(k, l)$.

removing the vertex of degree $k_{2}+1$ in $T$. By interlacing we have $\lambda_{2}(T) \leq \lambda_{1}\left(T^{\prime}\right)$. Since $k_{1} \geq 3$, we may apply the result given before Theorem 2.3 to get that $\lambda_{1}\left(T^{\prime}\right)$ is less than the largest root of

$$
\frac{1}{2}\left(x+\sqrt{x^{2}-4}\right) P_{K_{1, k_{1}}}(x)-P_{k_{1} K_{1}}(x),
$$

that is the largest root of

$$
\frac{1}{2}\left(x+\sqrt{x^{2}-4}\right)\left(x^{2}-k_{2}\right)-x .
$$

Setting $x=\sqrt{k_{1}+2}$, the last expression becomes equal to $\sqrt{k_{1}-2}$, i.e. it is positive in this point, which implies $\lambda_{1}\left(T^{\prime}\right)<\sqrt{k_{1}+2}$.

On the other hand, using interlacing once again, we get $\lambda_{2}(S) \geq \sqrt{k_{1}+2}$, which completes the proof.

In other words, in the set of generalized double comets with fixed internal path $P_{l}$, the minimal spectral gap is attained when the cocliques on $k_{1}$ and $k_{2}$ vertices are as equal as possible.

In order to compute the spectral gap of an arbitrarily large double comet, we derive formulas for computing its largest and second largest eigenvalue. The following two results may be viewed as a counterpart to [13, Proposition 2.4].

Theorem 2.4. If $\lambda_{1}(C(k, l))>2$, then $\lambda_{1}(C(k, l))$ is equal to $2 \cosh (2 t)$, where $t$ is the unique positive root of

$$
\left(e^{2 t(l-1)}+1\right) k-e^{-4 t}\left(e^{4 t}+1\right)\left(e^{2 t(l+1)}+1\right)=0 .
$$

Proof. Let the vertices of $C(k, l)$ be labeled $1,2, \ldots, 2 k+l$ in natural order (see Fig. 1), and let an eigenvector associated with $\lambda_{1}=\lambda_{1}(C(k, l))$ be denoted $x=\left(x_{1}, x_{2}, \ldots, x_{n}\right)^{T}$. Then we have

$$
\lambda_{1} x_{i}=\sum_{j \sim i} x_{j} \quad(1 \leq i \leq 2 k+l) .
$$

Using (2.2), we derive (2.1) in the following way. First, using the symmetry of $C(k, l)$, we may assume that $x_{1}=x_{2}=\cdots=x_{k}=x_{k+l+1}=x_{k+l+2}=\cdots=x_{2 k+l}$ (cf. [3]). Since the coordinates of the eigenvector $x$ are of the same sign and the eigenvector itself is determined up to a multiplicative constant, we may take $x_{1}=\lambda_{1}$. Then, we have 


$$
x_{k+1}=x_{k+l}=\lambda_{1}^{2} .
$$

For $0 \leq i \leq l-1$, using (2.2), we arrive at the following system of recurrence equations

$$
x_{k+i}-\lambda_{1} x_{k+i+1}+x_{k+i+2}=0 \quad(1 \leq i \leq l-1) .
$$

The former equality, that is (2.3), may be regarded as a boundary condition for (2.4). Solving the related characteristic equation $t^{2}-\lambda_{1} t+1=0$, we get $t_{1}=\frac{1}{t_{2}}=\frac{\lambda_{1}+\sqrt{\lambda_{1}^{2}-4}}{2}$, which yields

$$
x_{k+i+1}=c_{1} t_{1}^{k+i+1}+c_{2} t_{1}^{-(k+i+1)} .
$$

Setting $i=k+2$ and $i=k+l-1$ in (2.2), we deduce that

$$
x_{k+2}=x_{k+l-1}=\lambda_{1}\left(\lambda_{1}^{2}-k\right) .
$$

Next, using the first, and then the second equality in the last chain, we get $c_{2}=c_{1} t_{1}^{2 k+l+1}$ and $c_{1}=\frac{\lambda_{1}\left(\lambda_{1}^{2}-k\right)}{t_{1}^{k+2}+t_{1}^{k+l-1}}$, respectively. Substituting these expressions in (2.5) and using our boundary condition, we get

$$
\lambda_{1}\left(t_{1}^{k+2}+t_{1}^{k+l-1}\right)=\left(\lambda_{1}^{2}-k\right)\left(t_{1}^{k+1}+t_{1}^{k+l}\right) .
$$

Finally, using the obtained expression for $t_{1}$ and setting $\lambda_{1}=2 \cosh (2 t)$, after a straightforward computation we arrive at (2.1).

A simple analysis shows that the equation (2.1) has a positive real root. Moreover, it must be unique, because otherwise we would have two non-collinear eigenvectors corresponding to a simple eigenvalue $\lambda_{1}$. This observation completes the proof.

Our next result is obtained in the same way. We omit the proof and refer the reader to the previous theorem and [13].

Theorem 2.5. If $\lambda_{2}(C(k, l))>2$, then $\lambda_{2}(C(k, l))$ is equal to $2 \cosh (2 t)$, where $t$ is the unique positive root of

$$
\left(e^{4 t}-e^{2 t(l+1)}\right)(k-1)+e^{2 t(l+3)}-1=0 .
$$

The last two theorems enable us to compute the spectral gap of an arbitrary double comet. All what we need is to find the positive roots of (2.1) and (2.6) by means of numerical computation. In this way we get the trees with extremely small spectral gaps. For example, we have the following results for double comets determined by parameters $(k, l)$ :

\begin{tabular}{ccccccc}
\hline$(k, l)$ & $(10,10)$ & $(50,10)$ & $(100,10)$ & $(4,10)$ & $(4,50)$ & $(4,100)$ \\
$\delta \approx$ & 0.0001 & $1.7 \cdot 10^{-7}$ & $1.0 \cdot 10^{-8}$ & 0.0073 & $2.1 \cdot 10^{-12}$ & $2.5 \cdot 10^{-24}$ \\
\hline
\end{tabular}

So, Conjecture 2.1 remains open. If, for some $n$ there exists a tree which is not a double comet but has the minimal spectral gap then, by the previous computation, its spectral gap is very close to zero. Moreover, its second largest eigenvalue must be simple, as proved in our next statement. 
Theorem 2.6. If $T$ is a graph with minimal spectral gap in the set of trees of order $n$, then $\lambda_{2}(T)>\lambda_{3}(T)$.

Proof. Assume to the contrary. Since $T$ is a tree, it contains a vertex with two hanging paths attached. If these paths are $P_{n_{1}}$ and $P_{n_{2}}$ where, say $n_{1} \geq n_{2} \geq 2$, then let $T^{\prime}$ be the tree obtained by the relocation of the endvertex of $P_{n_{2}}$ to the end of $P_{n_{1}}$. It is known from numerous literature, see for example [14, Lemma 1.29(i)], that $\lambda_{1}(T)>\lambda_{1}\left(T^{\prime}\right)$. Next, since $\lambda_{2}(T)=\lambda_{3}(T)$, by interlacing, we get $\lambda_{2}(T) \leq \lambda_{2}\left(T^{\prime}\right)$. Altogether, $\delta(T)>\delta\left(T^{\prime}\right)$, a contradiction.

Recall that, according to the computer search, for $n \leq 20$, there is a unique tree that minimizes the spectral gap. So, there is another question: is such a tree unique for all $n$ ? A contribution to this question may be given by considering cospectrality of double comet graphs. It is not difficult to see that no every double comet has the unique spectrum (an easy exercise for the reader: show that $C(2, l)$ is cospectral with $\left.C_{4} \cup P_{l}\right)$. However, connected graphs that are cospectral with double comets are more interesting in our context. Here we provide the following result.

Theorem 2.7. The double comet $C(k, 2)$ is cospectral with a connected graph if and only if $k=t^{2}-t+1$, for $t \in \mathbb{N}$. The spectrum of a double comet $C(k, l), 3 \leq l \leq 5$, is unique in the set of connected graphs.

Sketch proof. Observe that cospectral graphs have equal orders and sizes, and therefore if a connected graph is cospectral with a double comet, then such a graph must be a tree. Next, recall from [6] that the multiplicity of zero (also known as nullity) in the spectrum of a tree is $n-4$ if and only if that tree is a generalized double comet $C^{*}\left(k_{1}, k_{2}, l\right)$, for $2 \leq l \leq 3$. Similarly, the trees of nullity $n-6$ are those illustrated in Fig. 2. Clearly, double comets specified in this theorem are covered by these sets of trees, and so to consider their cospectrality we need to compare their spectra with spectra of trees with equal number of zero eigenvalues.

For $l=2$, the possible candidates are the mentioned two generalized double comets with nullity equal to $n-4$. Recall (from the proof of Theorem 2.3) that the characteristic polynomial of $C^{*}\left(k_{1}, k_{2}, 2\right)$ is:

$$
P_{C^{*}\left(k_{1}, k_{2}, 2\right)}(x)=x^{k_{1}+k_{2}-2}\left(x^{4}-\left(k_{1}+k_{2}+1\right) x^{2}+k_{1} k_{2}\right) .
$$

Also, using the same method, we compute

$$
P_{C^{*}\left(k_{1}, k_{2}, 3\right)}(x)=x^{k_{1}+k_{2}-1}\left(x^{4}-\left(k_{1}+k_{2}+2\right) x^{2}+k_{1} k_{2}+k_{1}+k_{2}\right) .
$$

By setting $k_{1}=k_{2}=k$ in the first polynomial, we get the characteristic polynomial of $C(k, 2)$. Next, comparing the characteristic polynomials of $C(k, 2)$ and $C^{*}\left(k_{1}, k_{2}, 2\right)$, we get $k_{1}+k_{2}=k$ and $k_{1} k_{2}=k^{2}$, which implies $k_{1}=k_{2}$, i.e. the only solution is obtained when the corresponding trees are isomorphic.

Taking into account $C^{*}\left(k_{1}, k_{2}, 3\right)$, we get $k_{1}+k_{2}=2 k-1$ and $k_{1} k_{2}=(k-1)^{2}$, which means that $k_{1}$ and $k_{2}$ are the solutions of $s^{2}-(2 k-1) s+(k-1)^{2}=0$, i.e. they are of the form $\frac{2 k-1 \pm \sqrt{4 k-3}}{2}$. Since we are interested in integral solutions, $4 k-3$ must 

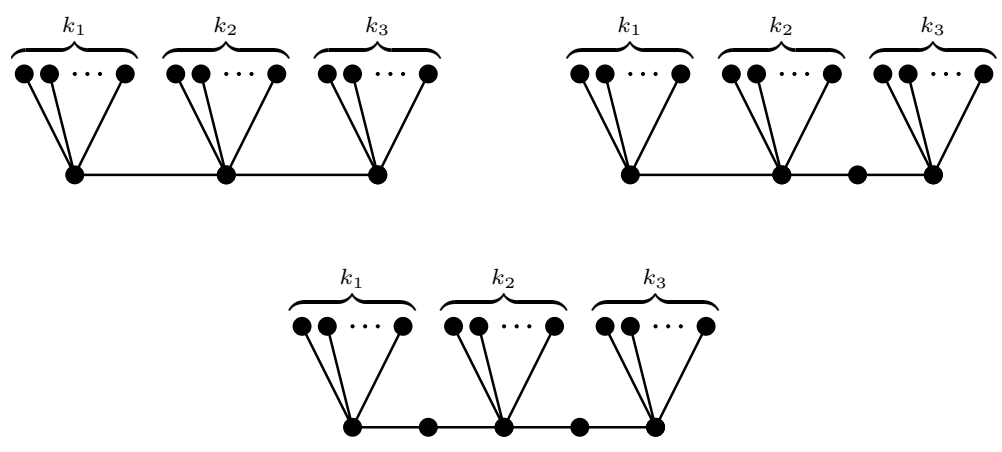

Figure 2: Trees of nullity $n-6$.

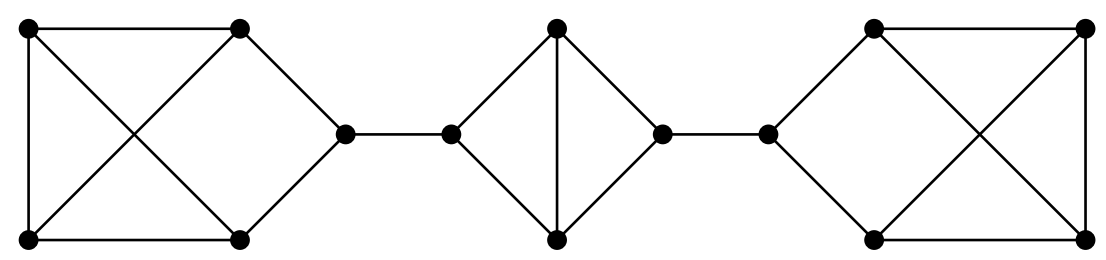

Figure 3: A regular graph whose spectral gap is smaller than the spectral gap of the corresponding path.

be a perfect square (clearly, an odd integer). Setting $4 k-3=(2 t-1)^{2}, t \in \mathbb{N}$, we get $k=t^{2}-t+1$. In addition, each $k$ of this form gives two distinct positive values for $k_{1}$ and $k_{2}$, and we are done.

The cases $l \in\{3,4,5\}$ are considered in exactly the same way, i.e. by comparing the corresponding characteristic polynomials and, in some situations, by reducing the procedure by using theoretic reasoning based on more sophisticated results like eigenvalue interlacing. In all cases we get that the double comet under consideration is not cospectral with any of possible candidates, and the proof follows.

So, in general case, a double comet may be cospectral with another graph. Even more, it may occur that this graph is connected (and then, it is also a tree). On the other hand, in our computational results double comets that are cospectral with other trees do not appear in the role of those with minimal spectral gap.

\section{Comparing the spectral gap of paths and regular graphs}

It is proved in [13] that the spectral gap of the path $P_{n}$ is always less than the spectral gap of the cycle $C_{n}$. In other words, the spectral gap of any connected 2-regular graph is greater than $\delta\left(P_{n}\right)$. Perhaps surprisingly, but a similar conclusion cannot be deduced for all regular graphs. A counterexample is the regular graph illustrated in Fig 3. Its spectral gap is close to 0.105 , while we simultaneously have $\delta\left(P_{14}\right) \approx 0.129$ (both values are rounded to three decimal places).

If the vertex degree is sufficiently large, then we have the following result. 
Theorem 3.1. Let $G$ be a connected $r$-regular graph of order $n(n \geq 3)$. If $r \geq 22$, then

$$
\delta(G)>\delta\left(P_{n}\right)
$$

Proof. If $n \leq 2 r+1$, then

$$
\lambda_{2}(G)=-\lambda_{n}(\bar{G}) \leq \lambda_{1}(\bar{G})=n-r-2,
$$

and so $\delta(G)=\lambda_{1}(G)-\lambda_{2}(G) \geq r-(n-r-2) \geq 1$. On the other hand, $\delta\left(P_{n}\right)<1$ holds for any $n \geq 5$, while the cases $n=3$ and $n=4$ are resolved easily.

Let now $n \geq 2 r+2$. Considering $\delta\left(P_{n}\right)$ and using the Taylor series, we get

$$
\begin{aligned}
\delta\left(P_{n}\right)= & 2\left(\cos \frac{\pi}{n+1}-\cos \frac{2 \pi}{n+1}\right) \\
< & 2\left(1-\frac{1}{2}\left(\frac{\pi}{n+1}\right)^{2}+\frac{1}{4 !}\left(\frac{\pi}{n+1}\right)^{4}-1\right. \\
& \left.+\frac{1}{2}\left(\frac{2 \pi}{n+1}\right)^{2}-\frac{1}{4 !}\left(\frac{2 \pi}{n+1}\right)^{4}+\frac{1}{6 !}\left(\frac{2 \pi}{n+1}\right)^{6}\right) \\
= & 2\left(\frac{3}{2}\left(\frac{\pi}{n+1}\right)^{2}-\frac{15}{4 !}\left(\frac{\pi}{n+1}\right)^{4}+\frac{1}{6 !}\left(\frac{2 \pi}{n+1}\right)^{6}\right) \\
= & 3\left(\frac{\pi}{n+1}\right)^{2}\left(1-\frac{5}{12}\left(\frac{\pi}{n+1}\right)^{2}+\frac{2^{3}}{5 \cdot 3^{3}}\left(\frac{\pi}{n+1}\right)^{4}\right) \\
< & 3\left(\frac{\pi}{n+1}\right)^{2} .
\end{aligned}
$$

We next use the inequalities for regular graphs, $\delta \geq \frac{4}{n D}\left[\right.$ [7] and $D \leq 3\left\lfloor\frac{n}{r+1}\right\rfloor-1$ [1], where $D$ stands for the diameter. We compute

$$
\begin{aligned}
\delta(G) & \geq \frac{4}{n D} \geq \frac{4}{n\left(3\left\lfloor\frac{n}{r+1}\right\rfloor-1\right)} \geq \frac{4}{n\left(3\left(\frac{n}{r+1}\right)-1\right)} \\
& =\frac{4(r+1)}{n(3 n-r-1)}>\frac{4(r+1)}{3 n^{2}}
\end{aligned}
$$

Since, for $r \geq 22$ it holds $3\left(\frac{\pi}{n+1}\right)^{2}<\frac{4(r+1)}{3 n^{2}}$, we get the assertion.

\section{Bounds for spectral gap}

In this section we give some bounds on $\delta(G)$. We start with the following lemma.

Lemma 4.1. Given a connected graph $G$, let $K$ denote its proper subgraph isomorphic to either a complete graph $K_{p}$ or a complete bipartite graph $K_{p, q}$. If $H$ is a graph obtained from $G$ by deleting all edges belonging to $K$, then

$$
\lambda_{2}(G) \leq \lambda_{1}(H)
$$


Proof. Assume that $G$ has $n$ vertices, and $K$ has $k$ vertices. Since $K$ is a proper subgraph, $k<n$ must hold. If $A$ is the adjacency matrix of $K \cup(n-k) K_{1}$ and $B$ is the adjacency matrix of $H$ then, by applying the Courant-Weyl inequality $\lambda_{2}(A+B) \leq \lambda_{2}(A)+\lambda_{1}(B)$ (cf. [2, Theorem 1.3.5]), we get

$$
\lambda_{2}(G) \leq \lambda_{2}\left(K \cup(n-k) K_{1}\right)+\lambda_{1}(H)=0+\lambda_{1}(H) .
$$

In what follows, we use the following upper bound for $\lambda_{1}^{k}$ ( $k$ being a positive integer) in terms of the clique number $\omega$ and the number of $k$-walks $w_{k}$ in $G$ [10],

$$
\lambda_{1}^{k} \leq \frac{\omega-1}{\omega} w_{k} .
$$

Theorem 4.2. For a connected graph $G$ with $n$ vertices, $m$ edges, and the clique number $\omega(\omega \geq 3)$,

$$
\delta \geq \omega-1-\sqrt{(\omega-2)\left(\frac{2 m}{\omega-1}-\omega\right)} .
$$

Proof. Setting $k=2$ in (4.1), we get $\lambda_{1}^{2} \leq 2 \frac{\omega-1}{\omega} m$. Deleting the edges belonging to a largest clique of $G$ and using Lemma 4.1, we get

$$
\lambda_{2}(G)^{2} \leq 2 \frac{\omega-2}{\omega-1}\left(m-\left(\begin{array}{l}
\omega \\
2
\end{array}\right)\right)
$$

or

$$
\lambda_{2}(G) \leq \sqrt{(\omega-2)\left(\frac{2 m}{\omega-1}-\omega\right)} .
$$

On the other hand, $\lambda_{1}(G) \geq \omega-1$, and we get the assertion.

Analyzing the lower bound (4.2), we get $\omega-1-\sqrt{(\omega-2)\left(\frac{2 m}{\omega-1}-\omega\right)} \geq 0$ whenever

$$
m \leq \frac{1}{2}+\omega(\omega-1)+\frac{1}{2 \omega-4} .
$$

In other words, this lower bound gives a non-trivial result only if the above inequality (on $m$ ) holds. Moreover, although the previous theorem is easily proved, the obtained inequality can give a good estimate in some cases. If we consider the graph $G$ obtained from the complete graph $K_{p}$ by attaching $k$ pendant edges at one of its vertices then (4.2) gives $\delta \leq p-1-\sqrt{k \frac{p-2}{p-1}}$. Setting $p=10$ and $k=1$ we get $\delta \approx 8.572$. The deviation form the exact value is close to 0.390 .

In the following theorem we provide more lower bounds on $\delta$. The first of them is based on the following inequality for a graph with $n$ vertices, $m$ edges and minimal vertex degree $d_{\min }[5,9]$,

$$
\lambda_{1} \leq \frac{d_{\min }-1+\sqrt{\left(d_{\min }+1\right)^{2}+4\left(2 m-d_{\min } n\right)}}{2}
$$


Theorem 4.3. Let $G$ be a connected $r$-regular graph with $n$ vertices and let $H$ be obtained form $G$ by deleting

(a) all edges belonging to a clique $K_{p}(p<n)$,

(b) all edges belonging to a proper induced subgraph $K_{p, q}(p \leq q)$,

then

(a) $\delta(G) \geq \frac{r+p-\sqrt{4 n(p-1)+(r+2)^{2}-3 p^{2}-2 r p}}{2}$,

(b) $\delta(G) \geq \frac{r+q+1-\sqrt{4 n q+(r-q+1)^{2}-8 p q}}{2}$ if $H$ is connected, and

$\delta(G) \geq \frac{r+p+1-\sqrt{4 n p+(r-p+1)^{2}-8 p q-4 p(r-p)}}{2}$ if $H$ is disconnected.

Proof. Consider (a). By Lemma 4.1, $\lambda_{2}(G) \leq \lambda_{1}(H)$. The graph $H$ has $n$ vertices, $\frac{r n}{2}-\left(\begin{array}{l}p \\ 2\end{array}\right)$ edges, and the minimal vertex degree in $H$ is $r-p+1$. Using the inequality (4.3), we get

$$
\lambda_{2}(G) \leq \lambda_{1}(H) \leq \frac{r-p+\sqrt{4 n(p-1)+(r+2)^{2}-3 p^{2}-2 r p}}{2},
$$

and since $\lambda_{1}(G)=r$, we get the assertion.

Consider now (b). If $H$ is connected then it has $n$ vertices, $\frac{r n}{2}-p q$ edges, and the minimal vertex degree in $H$ is $r-q$. Using (4.3), we get the assertion. If $H$ is disconnected then $p<q=r$, and it contains $p$ isolated vertices. Excluding these vertices, we get the graph with $n-p$ vertices and minimal vertex degree $r-p$. The desired inequality is again obtained by using the same argument.

Here is an upper bound for a special class of regular graphs.

Theorem 4.4. Let $G$ be an $r$-regular graph of order $n$. If $\bar{G}$ is triangle free, then

$$
\delta(G) \leq n\left(2-\frac{n}{r+1}\right) .
$$

Equality holds if $G$ is isomorphic to $K_{\frac{n}{2}, \frac{n}{2}}$.

Proof. We have $\lambda_{n}(\bar{G}) \leq-\frac{(n-r-1)^{2}}{r+1}$ (cf. [8]), and thus $\lambda_{2}(G)=-1-\lambda_{n}(\bar{G}) \geq-1+$ $\frac{(n-r-1)^{2}}{r+1}$. Now, $\delta(G)=r-\lambda_{2}(G)$ would be less than or equal to $r+1-\frac{(n-r-1)^{2}}{r+1}=$ $n\left(2-\frac{n}{r+1}\right)$ whenever this number is non-negative, i.e. whenever $r \geq \frac{n-2}{2}$. On the other hand, if $\bar{G}$ is triangle-free, then $\lambda_{1}(\bar{G}) \leq \sqrt{m(\bar{G})}$ (see [11]), i.e. $n-r-1 \leq \sqrt{\frac{n(n-r-1)}{2}}$, which again gives $r \geq \frac{n-2}{2}$. Hence, inequality (4.4) holds for any graph specified in the theorem.

The equality is verified by direct computation. 


\section{References}

[1] L. Caccetta and W. F. Smyth, Graphs of maximum diameter, Discrete Math. 102 (1992), 121141.

[2] D. Cvetković, P. Rowlinson and S. Simić, An Introduction to the Theory of Graph Spectra, Cambridge University Press, Cambridge, 2010.

[3] A. J. Hoffman, On limit points of spectral radii of non-negative symmetric integral matrices, in: Y. Alavi, D. R. Lick and A. T. White (eds.), Graph Theory and Applications, Springer, Berlin, 1972, 165-172.

[4] A. J. Hoffman and J. H. Smith, On the spectral radii of topologically equivalent graphs, in: M. Fiedler (ed.), Recent Advances in Graph Theory, Academia Praha, Prague, 1975, 273-281.

[5] Y. Hong, J.-L. Shu and K. Fang, A sharp upper bound of the spectral radius of graphs, J. Combin. Theory B 81 (2001), 177-183.

[6] S. Li, On the nullity of graphs with pendant vertices, Linear Algebra Appl. 429 (2008), 16191628.

[7] B. Mohar, The Laplacian spectrum of graphs, in: Y. Alavi, G. Chatrand, O.R. Oellermann and A.J. Schwenk (eds.), Graph Theory, Combinatorics and Applications, Wiley, New York, 1991, 871-898.

[8] V. Nikiforov, More spectral bounds on the clique and independence numbers, J. Combin. Theory $B 99$ (2009), 819-826.

[9] V. Nikiforov, Some inequalities for the largest eigenvalue of a graph, Comb. Probab. Comput. 11 (2002), 179-189.

[10] V. Nikiforov, Walks and the spectral radius of graphs, Linear Algebra Appl. 418 (2006), 257268.

[11] E. Nosal, Eigenvalues of Graphs, Master thesis, Univesity of Calgary, Calgary, 1970.

[12] S. K. Simić, E.M. Li Marzi and F. Belardo, On the index of caterpillars, Discrete Math. 308 (2008), 324-330.

[13] Z. Stanić, Graphs with small spectral gap, Electron. J. Linear Algebra 26 (2013), 417-432.

[14] Z. Stanić, Inequalities for Graph Eigenvalues, Cambridge University Press, Cambridge, 2015. 